\title{
Gesellschaft für Unterhaltungs- und Kommunikationselektronik - gfu
}

Elf führende Unternehmen der Unterhaltungselektronik haben 1973 die Gesellschaft zur Förderung der Unterhaltungselektronik gegründet. Ihre Ziele sind die Veranstaltung der Internationalen Funkausstellung Berlin, deren Rhythmus ab 2006 von zweijährlich auf jährlich geändert wurde, und die Information der Öffentlichkeit über die Entwicklung der Unterhaltungselektronik. Seit 1986 lautet der Name Gesellschaft für Unterhaltungs- und Kommunikationselektronik (gfu) $\mathrm{mbH}$.

Gesellschafter sind zwölf Mitgliedsfirmen des Fachverbandes Consumer Electronics im Zentralverband Elektrotechnik- und Elektronikindustrie (ZVEI) e. V. Die gfu veröffentlicht auf ihrer homepage (www.gfu.de) regelmäßig Daten über die Marktentwicklung für Unterhaltungs- und Kommunikationselektronik.

\section{Gewalt in den Medien}

Gewalt als beabsichtigte physische oder psychische Schädigung von Personen, Lebewesen oder Sachen wird in der öffentlichen Kommunikation häufig zum Thema gemacht. Schon die Geschichte von Kain und Abel ist ein Beispiel dafür, dass gewaltsam ausgetragene Konflikte mitteilenswert sind.

Da Normverstöße und Gewalttaten beim Publikum hohe $\rightarrow$ Aufmerksamkeit erlangen, sind sie auch als Bestandteil fiktionaler Medieninhalte, vom Roman bis hin zum Computerspiel, weit verbreitet. Angesichts der ständigen Präsenz von Gewaltdarstellungen im $\rightarrow$ Film, im $\rightarrow$ Fernsehen und in Computerspielen ( $\rightarrow$ Bildschirmspiele) wird nach möglichen Auswirkungen solcher Darstellungen gefragt. Im Hinblick auf Kinder und Jugendliche gibt es Befürchtungen, dass sie u. a. durch Gewaltdarstellungen in ihrer persönlichen Entwicklung gefährdet werden können. Im Jugendschutzgesetz und im JugendmedienschutzStaatsvertrag sind deshalb u. a. solche Angebote untersagt, die Gewalttätigkeiten verherrlichen oder verharmlosen ( $\rightarrow$ Jugendschutz). 